University of New Hampshire

University of New Hampshire Scholars' Repository

Space Science Center

Institute for the Study of Earth, Oceans, and

Space (EOS)

2000

\title{
First results of a study of TeV emission from GRBs in Milagrito
}

\author{
J E. McEnery \\ University of Utah \\ R Atkins \\ University of Utah \\ W Benbow \\ University of California - Santa Cruz \\ D Berley \\ University of Maryland - College Park \\ M L. Chen \\ University of Maryland - College Park \\ Follow this and additional works at: https://scholars.unh.edu/ssc \\ See next page for additional authors \\ Part of the Astrophysics and Astronomy Commons
}

\section{Recommended Citation}

First results of a study of TeV emission from GRBs in Milagrito McEnery, J. E. and Atkins, R. and Benbow, W. and Berley, D. and Chen, M. L. and Coyne, D. G. and Dingus, B. L. and Dorfan, D. E. and Ellsworth, R. W. and Evans, D. and Falcone, A. and Fleysher, L. and Fleysher, R. and Gisler, G. and Goodman, J. A. and Haines, T. J. and Hoffman, C. M. and Hugenberger, S. and Kelley, L. A. and Leonor, I. and McConnell, M. and McCullough, J. F. and Miller, R. S. and Mincer, A. I. and Morales, M. F. and Nemethy, P. and Ryan, J. M. and Shen, B. and Shoup, A. and Sinnis, C. and Smith, A. J. and Sullivan, G. W. and Tumer, T. and Wang, K. and Wascko, M. O. and Westerhoff, S. and Williams, D. A. and Yang, T. and Yodh, G. B., AIP Conference Proceedings, 515, 243-248 (2000), DOI:http://dx.doi.org/10.1063/1.1291374

This Conference Proceeding is brought to you for free and open access by the Institute for the Study of Earth, Oceans, and Space (EOS) at University of New Hampshire Scholars' Repository. It has been accepted for inclusion in Space Science Center by an authorized administrator of University of New Hampshire Scholars' Repository. For more information, please contact Scholarly.Communication@unh.edu. 


\section{Authors}

J E. McEnery, R Atkins, W Benbow, D Berley, M L. Chen, D G. Coyne, B L. Dingus, D E. Dorfan, R W. Ellsworth, D Evans, A Falcone, L Fleysher, R Fleysher, G Gisler, J A. Goodman, T J. Haines, C M. Hoffman, S Hugenberger, L A. Kelley, I Leonor, Mark L. McConnell, J F. McCullough, R S. Miller, A I. Mincer, M F. Morales, P Nemethy, James M. Ryan, B Shen, A Shoup, C Sinnis, A J. Smith, G W. Sullivan, O T. Tumer, K Wang, M O. Wascko, S Westerhoff, D A. Williams, T Yang, and G B. Yodh 


\section{AIP $\mid$ proceedings}

\section{First results of a study of TeV emission from GRBs in Milagrito}

J. E. McEnery, R. Atkins, W. Benbow, D. Berley, M. L. Chen, D. G. Coyne, B. L. Dingus, D. E. Dorfan, R. W. Ellsworth, D. Evans, A. Falcone, L. Fleysher, R. Fleysher, G. Gisler, J. A. Goodman, T. J. Haines, C. M. Hoffman , S. Hugenberger, L. A. Kelley, I. Leonor, M. McConnell, J. F. McCullough, R. S. Miller, A. I. Mincer, M. F. Morales, P. Nemethy, J. M. Ryan, B. Shen, A. Shoup, C. Sinnis, A. J. Smith, G. W. Sullivan, T. Tumer, K. Wang, M. O. Wascko, S. Westerhoff, D. A. Williams, T. Yang, and G. B. Yodh

Citation: AIP Conference Proceedings 515, 243 (2000); doi: 10.1063/1.1291374

View online: http://dx.doi.org/10.1063/1.1291374

View Table of Contents: http://scitation.aip.org/content/aip/proceeding/aipcp/515?ver=pdfcov Published by the AIP Publishing

\section{Articles you may be interested in}

TeV observations of $\mathrm{X}$-ray binaries

AIP Conf. Proc. 515, 271 (2000); 10.1063/1.1291378

Detection of TeV gamma rays from Mrk 501 at high flaring state of activity in 1997 with the Tibet air shower array AIP Conf. Proc. 515, 139 (2000); 10.1063/1.1291358

Flux limits for TeV emission from AGNs

AIP Conf. Proc. 515, 91 (2000); 10.1063/1.1291349

TeV emission from PKS2155-304

AIP Conf. Proc. 515, 86 (2000); 10.1063/1.1291348

First results of a study of TeV emission from GRBs in Milagrito

AIP Conf. Proc. 510, 543 (2000); 10.1063/1.1303263 


\title{
First Results of a Study of TeV Emission from GRBs in Milagrito
}

\author{
J. E. McEnery ${ }^{1}$, R. Atkins ${ }^{1}$, W. Benbow ${ }^{2}$, D. Berley ${ }^{3,10}$, \\ M.L. Chen ${ }^{3,11}$, D.G. Coyne ${ }^{2}$, B.L. Dingus ${ }^{1}$, D.E. Dorfan ${ }^{2}$, \\ R.W. Ellsworth ${ }^{5}$, D. Evans ${ }^{3}$, A. Falcone ${ }^{6}$, L. Fleysher ${ }^{7}$, R. Fleysher ${ }^{7}$, \\ G. Gisler ${ }^{8}$, J.A. Goodman ${ }^{3}$, T.J. Haines ${ }^{8}$, C.M. Hoffman ${ }^{8}$, \\ S. Hugenberger ${ }^{4}$, L.A. Kelley ${ }^{2}$, I. Leonor ${ }^{4}$, M. McConnell ${ }^{6}$, \\ J.F. McCullough ${ }^{2}$, R.S. Miller,6, A.I. Mincer ${ }^{7}$, M.F. Morales ${ }^{2}$, \\ P. Nemethy ${ }^{7}$, J.M. Ryan ${ }^{6}$, B. Shen ${ }^{9}$, A. Shoup ${ }^{4}$, C. Sinnis ${ }^{8}$, \\ A.J. Smith ${ }^{9}$, G.W. Sullivan ${ }^{3}$, T. Tumer ${ }^{9}$, K. Wang ${ }^{9}$, M.O. Wascko ${ }^{9}$, \\ S. Westerhoff ${ }^{2}$, D.A. Williams ${ }^{2}$, T. Yang ${ }^{2}$, G.B. Yodh ${ }^{4}$
}

(1) University of Utah, Salt Lake City, UT 84112, USA

(2) University of California, Santa Cruz, CA 95064, USA

(3) University of Maryland, College Park, MD 20742, USA

(4) University of California, Irvine, CA 92697, USA

(5) George Mason University, Fairfax, VA 22030, USA

(6) University of New Hampshire, Durham, NH03824, USA

(7) New York University, New York, NY10003, USA

(8) Los Alamos National Laboratory, Los Alamos, NM 87545, USA

(9) University of California, Riverside, CA 92521, USA

(10) Permanent Address: National Science Foundation, Arlington, VA ,22230, USA

(11) Now at Brookhaven National Laboratory, Upton, NY11973, USA

\begin{abstract}
Milagrito, a detector sensitive to $\gamma$-rays at $\mathrm{TeV}$ energies, monitored the northern sky during the period February 1997 through May 1998. With a large field of view and high duty cycle, this instrument was used to perform a search for $\mathrm{TeV}$ counterparts to $\gamma$-ray bursts. Within the Milagrito field of view $54 \gamma$-ray bursts at keV energies were observed by the Burst And Transient Satellite Experiment (BATSE) aboard the Compton Gamma-Ray Observatory. This paper describes the results of a preliminary analysis to search for $\mathrm{TeV}$ emission correlated with BATSE detected bursts. Milagrito detected an excess of events coincident both spatially and temporally with GRB 970417 a, with chance probability $2.8 \times 10^{-5}$ within the BATSE error radius. No other significant correlations were detected. Since 54 bursts were examined the chance probability of observing an excess with this significance in any of these bursts is $1.5 \times 10^{-3}$. The statistical aspects and physical implications of this result are discussed.
\end{abstract}

CP515, GeV-TeV Gamma Ray Astrophysics Workshop, edited by B. L. Dingus, et al. (C) 2000 American Institute of Physics 1-56396-938-6/00/\$17.00 


\section{OBSERVATIONS AND ANALYSIS}

Milagro, a new type of $\mathrm{TeV} \gamma$-ray observatory sensitive at energies above 100 $\mathrm{GeV}$, with a field of view of over one steradian and a high duty cycle, began operation in February 1999, near Los Alamos, NM. A predecessor of Milagro, Milagrito [5], operated from February 1997 to May 1998. During this time interval, 54 $\gamma$-ray bursts (GRBs) detected by BATSE [1] were within Milagrito's field of view (less than $45^{\circ}$ zenith angle).

A search was conducted in the Milagrito data for an excess of events above the cosmic-ray background coincident with each of these $\gamma$-ray bursts. For each burst, a circular search region was defined by the BATSE $90 \%$ confidence interval, which incorporates both the statistical and systematic position errors [2]. The size of this $90 \%$ confidence interval ranged from $4^{\circ}$ to $26^{\circ}$ for the 54 GRBs in the sample. The search region was tiled with an array of overlapping $1.6^{\circ}$ radius bins centered on a $0.2^{\circ} \times 0.2^{\circ}$ grid. This radius was derived from the measured angular resolution of Milagrito and was selected prior to the search. The number of events falling within each of the $1.6^{\circ}$ bins was tallied for the duration of the burst reported by BATSE. This duration is defined as the time required for BATSE to accumulate $90 \%$ of the $\gamma$-rays(T90). T90 ranged from 0.1 seconds to 195 seconds for the 54 bursts examined.

The angular distribution of background events on the sky was characterized using two hours of data surrounding each burst. This distribution was normalized to the number of events detected by Milagrito over the entire sky during the T90 interval $\left(N_{T 90}\right)$. The resulting background data were also binned in $1.6^{\circ}$ bins spaced $0.2^{\circ}$ apart. The Poisson probability that the excess of events in each $1.6^{\circ}$ bin was due to a background fluctuation was calculated and the bin with lowest probability was taken as the candidate position of a TeV $\gamma$-ray counterpart to the BATSE burst. The background and signal counts in this bin were used to calculate a fluence or fluence upper limit for each burst.

\section{RESULTS}

The flux sensitivity of Milagrito to $\gamma$-ray bursts depends on the zenith angle and duration of the burst, and on the instrument conditions at the time. During the lifetime of the Milagrito detector, data were taken with three different water depths $(0.9 \mathrm{~m}, 1.5 \mathrm{~m}$ and $2.0 \mathrm{~m})$. In addition, for the period February 1997 through the end of March 1997 a considerable amount of snow collected on the cover of the pond. Detector simulations were used to obtain effective area as a function of zenith angle for an assumed $\mathrm{E}^{-2.0}$ spectrum for each of these configurations. These were then used to calculate flux upper limits for each burst. Flux upper limits in the range $10^{-6}-10^{-8} \mathrm{\gamma} / \mathrm{cm}^{2} / \mathrm{s}$ were obtained for 53 of the 54 bursts in the sample. 


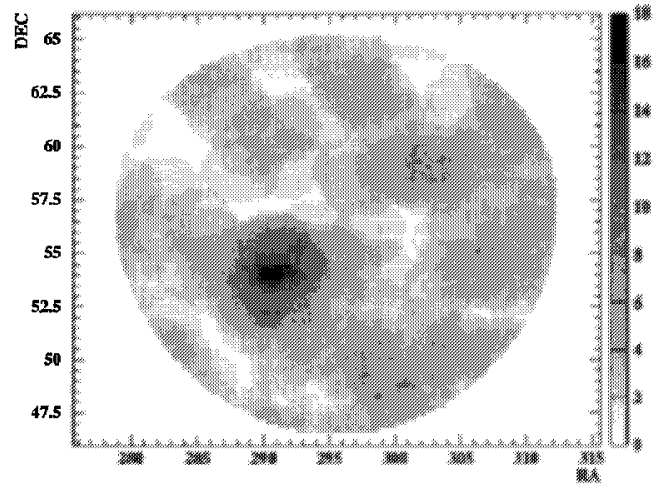

FIGURE 1. Number of events recorded by Milagrito during T90 in the BATSE error radius for GRB $970417 \mathrm{a}$, each bin contains the number of events detected by Milagrito within a 1.6 degree radius.
Of the 54 bursts one, GRB 970417a, shows a substantial excess above background in the $\mathrm{Mi}$ lagrito data. The BATSE detection of this burst is a weak burst with a fluence in the 50$300 \mathrm{keV}$ energy range of $1.5 \times 10^{-7}$ ergs $/ \mathrm{cm}^{2}$ and T90 of 7.9 seconds. BATSE determined the burst position to be $\mathrm{RA}=295.66^{\circ}, \mathrm{DEC}=$ $55.77^{\circ}$. The $90 \%$ positional uncertainty was $9.4^{\circ}$. The $1.6^{\circ}$ radius bin with the largest excess in the Milagrito data is centered at $\mathrm{RA}=289.89^{\circ}$ and $\mathrm{DEC}=54.0^{\circ}$, corresponding to a zenith angle of $21^{\circ}$. This position is $3.8^{\circ}$ away

from the position reported by BATSE; well within the BATSE 1-sigma position error $6.2^{\circ}$. The uncertainty in the position of the $\mathrm{TeV}$ candidate was determined by Monte-Carlo simulations to be approximately $0.5^{\circ}$. Figure 1 shows the number of counts in this search region for the array of $1.6^{\circ}$ bins. The bin with the largest excess has 18 events with an expected background of $3.46 \pm 0.11$. The Poisson probability for observing a signal at least this large due to a background fluctuation is $2.89 \times 10^{-8}$.

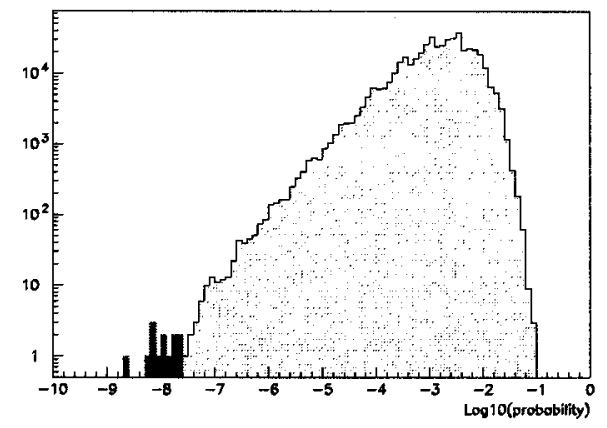

FIGURE 2. The distribution of minimum probabilities for the ensemble of simulated data-sets for GRB 970417a.
To obtain the significance of this result one must account for the size of the search region. The probability of obtaining the observed significance anywhere within the entire search region was determined by Monte Carlo simulations. A set of simulated signal maps was made by randomly drawing $N_{T 90}$ events from the background distribution. Each map was searched, as before, for a significant excess within the search region defined by BATSE. The probability of the observation in the actual data being due to a fluctuation in the background, after accounting for the size of the search region, is given by the ratio of the number of simulated data sets with probability less than that observed for the actual data to the total number of simulated data sets. The distribution of the probabilities for $4.65 \times 10^{6}$ simulated data sets is shown in figure 2; thirteen of which had Poisson probability less than $2.89 \times 10^{-8}$. We therefore 
find that the chance probability of such a detection within the entire $9.4^{\circ}$ search region for GRB 970417 a to be $2.8 \times 10^{-5}$. The probabilities for each of the other 53 bursts in the sample were obtained using the same method, the distribution of these probabilities, after correcting for the size of the search region, is shown in figure 3. The histogram on the left, plotted on a log-linear scale, illustrates the significance of the excess for GRB 970417a relative to the rest of the sample. The histogram on the right of this figure, plotted on a linear scale is flat, as expected. 54 bursts were examined. Therefore the chance probability of observing such a significant excess due to fluctuations in the background for any of these bursts is $1.5 \times 10^{-3}$.
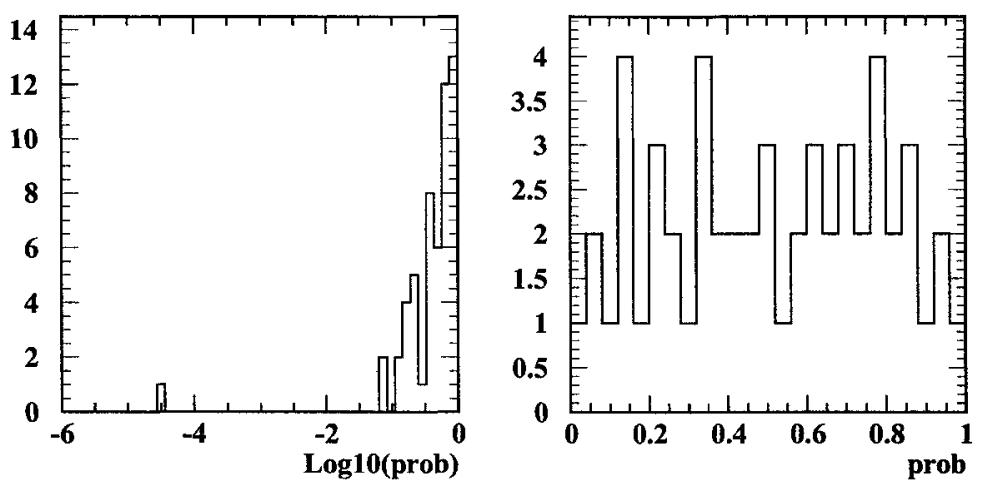

FIGURE 3. The distribution of probabilities, corrected for the size of the search region for the 54 GRBs in the sample, both plots show the same data with a linear and logarithmic scale for the $\mathrm{x}$-axis

Although the initial search was limited to T90, for GRB 970417a longer time intervals were also examined. To allow for the positional uncertainty of the excess observed by Milagrito, the radius of the search bin was increased to $2.2^{\circ}$ for this search. A search for $\mathrm{TeV} \gamma$-rays integrated over long time intervals of one hour, two hours and a day after the GRB start time did not show any significant excess. Lightcurves where the data are binned in intervals of one second and of T90 (7.9 s) are shown in figure 4. A preliminary analysis reveals no statistically compelling evidence for $\mathrm{TeV}$ afterflares.

\section{DISCUSSION}

If the observed excess of events in Milagrito is indeed associated with GRB 970417a then it represents the highest energy photons yet detected from a GRB in coincidence with the sub-MeV emission. The following discussion assumes that the excess observed by Milagrito was due to $\mathrm{TeV} \gamma$-rays from GRB 970417a. The $\mathrm{TeV}$ spectrum and maximum energy of emission is difficult to determine from Milagrito data [5]. Monte Carlo simulations of $\gamma$-ray initiated air showers show that the 

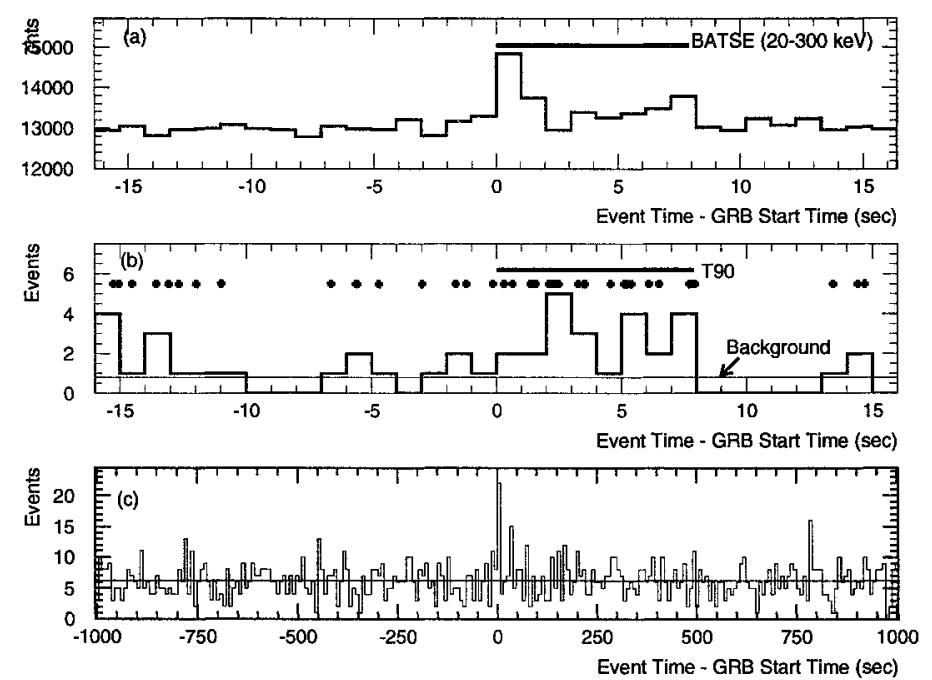

FIGURE 4. GRB 970417a: (a) The BATSE lightcurve, (b) Milagrito data within a $2.2^{\circ}$ radius of GRB 970417a integrated in 1 second bins, the crosses indicate the arrival times of the events and (c) integrated in bins of 7.9 seconds (T90) for 2000 seconds

effective area increases smoothly with energy, making the definition of an energy threshold ambiguous. Figure 5 shows the implied fluence of this observation as a function of upper cutoff energy for a range of power-law input spectra.

Some information about the energies of the events observed for GRB 970417a can be obtained by considering the response of the summed untriggered counting rate of the individual PMTs in Milagrito. Detector simulations of the effect on PMT counting rates of $\gamma$-ray induced air-showers indicate that these rates are more sensitive than the standard shower data at energies below a few hundred $\mathrm{GeV}$, but are only sensitive to very large fluxes [5]. No excess was observed in these rates, which implies that the air-showers detected by Milagrito were probably due to $\gamma$-rays at energies above several hundred $\mathrm{GeV}$.

High energy $\gamma$-rays from sources at cosmological distances will be absorbed via electron-positron pair production with infrared photons in the intergalactic medium. Several studies find that the opacity due to pair production for above $200 \mathrm{GeV}$ $\gamma$-rays exceeds one for redshifts larger than $0.3[3,4]$. Thus, if Milagrito has indeed detected high energy photons from GRB 990417a, it must be from a relatively nearby object.

\section{CONCLUSION}

An excess of events with chance probability $2.8 \times 10^{-5}$ coincident both spatially and temporally with the BATSE emission for GRB 970417a was observed by 


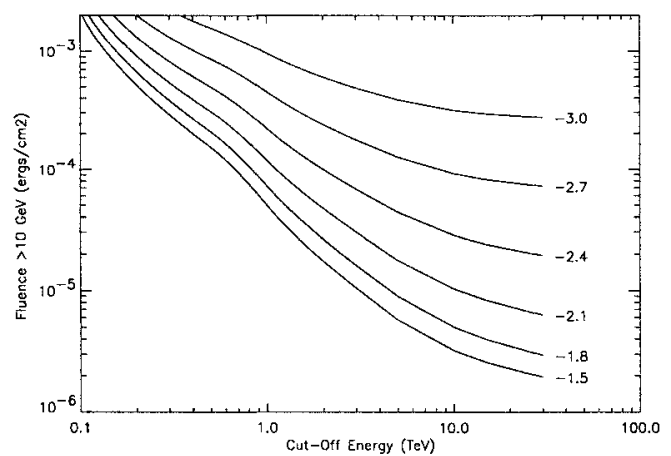

FIGURE 5. Implied fluences of this candidate for a range of assumed power-law spectra and high energy cutoffs (preliminary)

Milagrito. The chance probability that an excess of this significance would be observed from the entire sample of 54 bursts is $1.5 \times 10^{-3}$. The spectrum must extend with no cutoff to at least a few hundred $\mathrm{GeV}$. The inferred $\mathrm{TeV}$ fluence from this result at least an order of magnitude greater than the sub-MeV fluence and the emission extends to at least several hundred $\mathrm{GeV}$.

If the observed excess from GRB 970417a is not a fluctuation of the background, then a new class of $\gamma$-ray bursts bright at $\mathrm{TeV}$ energies may have been observed. A search for other coincidences with BATSE, to verify this result, will be continued with the current instrument, Milagro, which has increased sensitivity to $\mathrm{TeV} \gamma$-ray bursts.

\section{ACKNOWLEDGMENTS}

This research was supported in part by the National Science Foundation, the U.S. Department of Energy Office of High Energy Physics, the U.S. Department of Energy Office of Nuclear Physics, Los Alamos National Laboratory, the University of California, the Institute of Geophysics and Planetary Physics, The Research Corporation, and the California Space Institute.

\section{REFERENCES}

1. W. S. Paciesas et al., (Astro-Ph-9903205) (1999)

2. M. S. Briggs et al., Astrophys. J. Supp. 122(2), 503 (1999)

3. M.H. Salamon and F. W. Stecker, Astrophys. J. 493, 547 (1998).

4. J. R. Primack et al, Astroparticle Physics 11, 93 (1999).

5. R. Atkins et al., Nucl. Inst. and Methods (1999) (submitted). 